\title{
Soziale Arbeit dauerhaft sichern
}

\section{Bürger- und Gemeinschaftsstiftungen sind innovatives Fundraising mit Traditionsbildung}

\author{
Wolfgang Antes
}

\begin{abstract}
Annually, about 200 billion euro are bequeathed in Germany. Through the five years between 2000 and 2005 4,800 new foundations have been established. This shows that charitable organisations are increasingly recognising that the establishment of a foundation provides a sustainable instrument for fundraising. However, in many cases the respective organisations cannot manage the conceptual and legal obstacles. The concept of a citizens' or community foundation provides an interesting perspective.
\end{abstract}

Chaque année, près de 200 milliards d'euros sont légués en Allemagne. Déjà de l'année 2000 à l'année 2005 il y a eu création de 4800 nouvelles fondations. Cela montre également que les organisations caritatives ont compris à quel point la création d'une fondation pouvait constituer un instrument de collecte de fonds durable. Cependant, dans beaucoup de cas, les organisations ont du mal à maîtriser les obstacles conceptuels et juridiques. L'idée d'une fondation citoyenne ou communautaire apporte à ce titre une perspective intéressante.

Wolfgang Antes ist Geschäftsführer der Jugendstiftung Baden-

Württemberg.

E-Mail antes@jugendstiftung.de
Jährlich werden in Deutschland etwa 200 Milliarden Euro vererbt. Allein im Zeitraum von 2000 bis 2005 wurden 4.800 Stiftungen neu gegründet. Das zeigt, dass auch gemeinnützige Organisationen die Möglichkeiten zunehmend erkennen, die die Gründung einer Stiftung als nachhaltiges Fundraisinginstrument bietet. Oftmals scheitern aber gemeinnützige Organisationen an konzeptionellen und rechtlichen Hürden. Das Konzept der Bürger-oder Gemeinschaftsstiftung bietet hier eine interessante Perspektive.

In Abgrenzung zu herkömmlichen Stiftungen, bezeichnet der Begriff Bürgerstiftung, dass sich mehrere Personen, anstatt eines alleinigen Stifters, zusammenschließen, um gemeinsam eine Stiftung einzurichten. Die Gemeinschaftsstiftung bezeichnet eher den Sachverhalt, dass mehrere gemeinnützige Organisationen eine Stiftung als Verbund gemeinsam errichten und betreiben. Der Einfachheit halber wird im Folgenden der Begriff Gemeinschaftsstiftung verwendet und der Fokus auf die Kooperation von gemeinnützigen Organisationen gelegt. Diese haben gute Gründe, über die Errichtung einer gemeinsam betriebenen Stiftung nachzudenken:

- Sie möchten dauerhaft einen Arbeitsbereich absichern, der allein aus öffentlichen Mitteln nicht finanzierbar ist.

- Ein einzelner Träger kann finanziell oftmals eine Stiftungsgründung nicht bewältigen, da ein wesentliches Hindernis darin besteht, einen angemessenen Kapitalstock zu bilden.

- Für eine Stiftungsgründung sollten nach gängiger Rechtspraxis mindestens 50.000 Euro zur Verfügung stehen.

- Gleichzeitig muss jedoch der Ertrag, also die Rendite aus dem gebildeten Kapitalstock, die neu gegründete Stiftung in die Lage versetzen, ihrem Satzungsanspruch auch gerecht $\mathrm{zu}$ werden. Zum Beispiel wird sich eine Jugendkulturstiftung mit einem Kapital von 100.000 Euro und einer Jahresrendite von 5.000 Euro schwerlich Kulturstiftung Schleswig-Holstein nennen dürfen. Da hier das ambitionierte Ziel, die Jugendkultur in Schleswig-Holstein zu fördern, mit dem relativ bescheidenen Renditeertrag von 5.000 Euro nicht zu realisieren ist. Die Stiftungsaufsicht wird also keine Genehmigung geben. Anders sieht es aus, wenn besagte Kulturstiftung lediglich für einen Landkreis fördernd tätig sein will. Hierfür sind 5.000 Euro ein durchaus respektabler Förderungsbetrag. Dennoch ist es oftmals nicht einfach, für engagierte Einzelpersonen, gemeinnützige Vereine oder sonstige Initiativgruppen, die Anfangshürde von rund 50.000 Euro für den Erstkapitalstock zu nehmen.

- Die Errichtung und vor allem der Aufbau einer Kapitalstiftung erfordern langfristige, gut durchdachte konzeptionelle Planungen im Bereich Kommunikation, Public Relations und Netzwerkarbeit. Es ist gut möglich, dass sich diese Absicht im Verbund mit anderen Trägern und Partnern überhaupt erst nachhaltig organisieren lässt. Zum Beispiel bringt ein Verein, der ausschließlich aus einer Vielzahl von ehrenamtlichen Mitgliedern besteht, wichtige Kontaktmöglichkeiten ein, die durch die professionelle Struktur einer anderen Organisation ergänzt wird, die hier aber auf kein breites Mitgliedernetz zurückgreifen kann.

Mit anderen Worten: Das Konstrukt Gemeinschaftsstiftung bietet eine interessante Variante, Ziele, die dem Gemeinwohl dienen, in einer Region langfristig zu verfolgen und damit eine ebenso langfristige Fundraisingstrategie $\mathrm{zu}$ entwickeln.

Gleichzeitig ist damit eine Traditionsbildung verbunden, die nicht hoch genug eingeschätzt werden kann. Dies geschieht langsam, unmerklich. Wir sind es nicht gewohnt, langfristige Werte- und Tradi- 
tionsbildungen bewusst wahrzunehmen. Wie gebannt blicken wir auf »traditionsreiche" Unternehmungen, die auf einer scheinbar nicht rezipierbaren Wertebasis gewachsen sind und heute über ein großartiges Image verfügen. Genau das Gleiche ist heute und jetzt möglich! Und auch den Initiatoren von Harvard war in den ersten Jahren sicher nicht klar, welch herausragenden Effekt ihre Stiftung erlangen sollte.

Gemeinnützige Vereine erhalten mit einer Stiftung ein Instrument, mit der die Absicht unterstützt wird, Vermögen langfristig einzuwerben und nachhaltiger als bisher gemeinnützige Zwecke zu verfolgen (vgl. Abb. 1). Das Schaubild zeigt wie mehrere zustiftende, gemeinnützige Organisationen, gemeinsames Kapital in Form einer Stiftung Bürgerlichen Rechts aufbauen. Bei dieser Konstruktion fließt das Kapital in einen gemeinsamen Pool und geht darin auf.

Die Abbildung 1 zeigt aber auch bereits interessante Weiterentwicklungsmöglichkeiten: Ebenso gut ist es möglich, dass die gegründete Stiftung Bürgerlichen Rechts als "Stiftungsdach « fungiert und die einzelnen Beträge, die von den jeweiligen Organisationen eingebracht werden, sogenannte rechtlich nicht selbstständige Stiftungen zugewiesen werden und diese entsprechend zu benennen und auszuweisen sind.

Das klingt kompliziert, ist rechtlich aber einfach. Denn jeder Geldbetrag, der als Sondervermögen dazu bestimmt wird aus seinen Kapitalerträgen eine Förderaufgabe zu finanzieren, ist per Definition eine rechtlich unselbstständige Stiftung. Allerdings bedürfen rechtlich nicht selbstständige Stiftungen eines Treuhänders, der vertraglich abgesichert, die korrekte Kontrolle und Verwaltung des Vermögens garantiert. Treuhänder können eine private Person sein, eine soziale Organisation, eine Bank oder eben eine Stiftung Bürgerlichen Rechts. Abbildung 2 zeigt an einer fiktiven Konstruktion ein solches Beispiel. Hier wird deutlich, wie ganz unterschiedliche Organisationen in einer Region in eine gemeinsame Zielsetzung eingebunden werden.

Werfen wir einen Blick auf den Spenderund Stiftermarkt. Die Debatte um Bürgerbeteiligung und Partizipation junger Menschen in den letzten Jahren und Jahrzehnten hat gezeigt, dass immer mehr Menschen bereit sind, sich im Gemeinwesen zu beteiligen und dass sie bereit sind, hier grundsätzlich Verantwortung zu übernehmen. Das spiegelt sich auch in einer erhöhten Bereitschaft wieder, eigene Vermögensanteile zu stiften, das heißt abzugeben. So hat sich in den letzten Jahren die Anzahl der jährlich neu gegründeten Stiftungen ständig erhöht. Von 1980 bis 1989 gab es nur 1.576 Neugründungen. Diese Zahl wird heute in drei Jahren erreicht. Tendenz weiter steigend. In den nächsten Jahren steht ein Vermögen von einer Billion Euro zur Vererbung an. In den achtziger Jahren betrug das jährliche Erbschaftsvolumen noch etwa 50 Milliarden Euro. Jetzt sind es jährlich etwa 200 Milliarden Euro. Diese Situation ist in der Geschichte der Bundesrepublik ohne Beispiel.

Es mag zunächst eine ungewohnte Überlegung sein, aber für den gemein- nützigen Bereich und damit für den gesamten dritten Sektor wird es von wesentlicher Bedeutung sein, welcher Anteil dieser Summe in den nächsten Jahren und Jahrzehnten dauerhaft für eben diese Arbeit generiert und geworben werden kann.

Dabei sollte man sich von der Vorstellung lösen, dass alte und gebrechliche Menschen mangels eigener Erben davon überzeugt werden sollen, Großteile ihres Vermögens einem bestimmten gemeinnützigen Verein zu überschreiben. Das mag im Einzelfall so sein, ist hier aber nicht beabsichtigt. Viel wichtiger ist es, eine Kultur des verantwortungsbewussten Vererbens und der bewussten Traditionsbildung durch Testamentsgestaltung in der aktiven Generation der 40- bis 60Jährigen $\mathrm{zu}$ ermöglichen und hier ent-

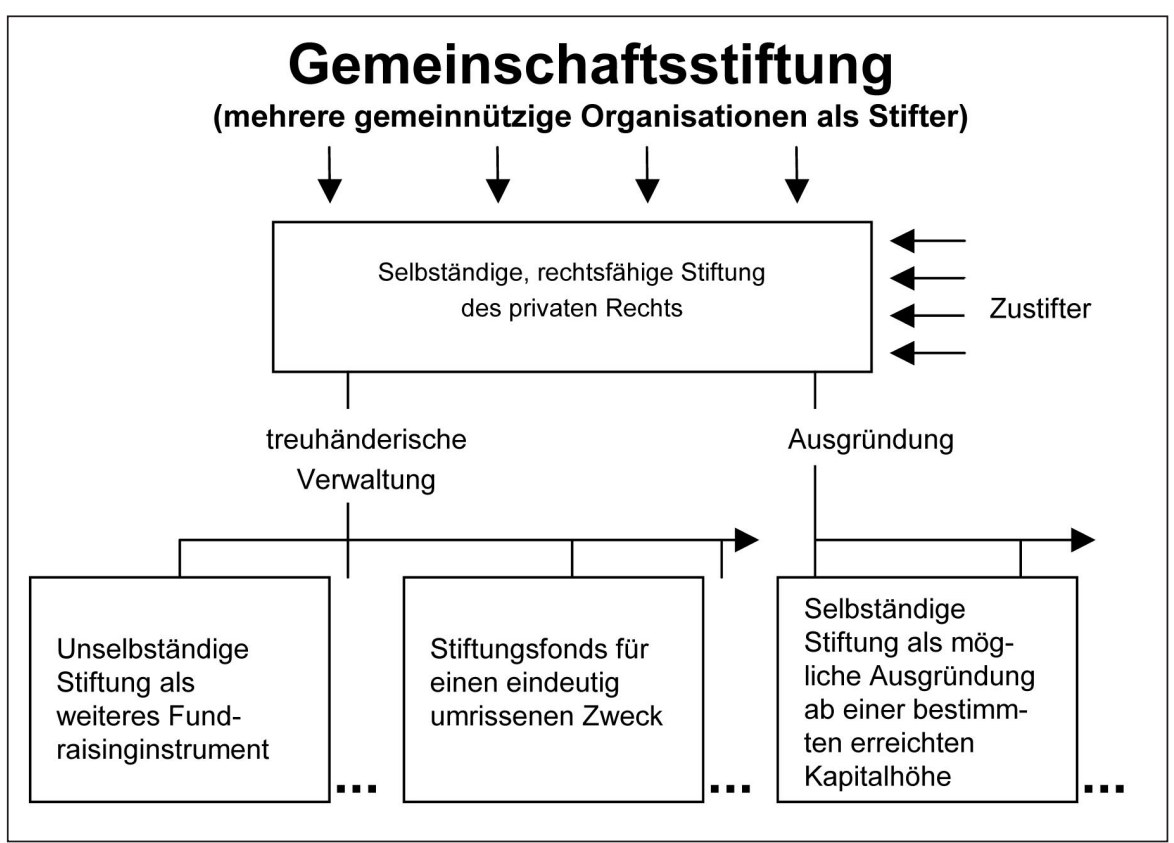

Abb. 1: In einer »Gemeinschaftsstiftung « können sich mehrere Einzelpersonen und Organisationen unter einem Dach zusammenfinden.

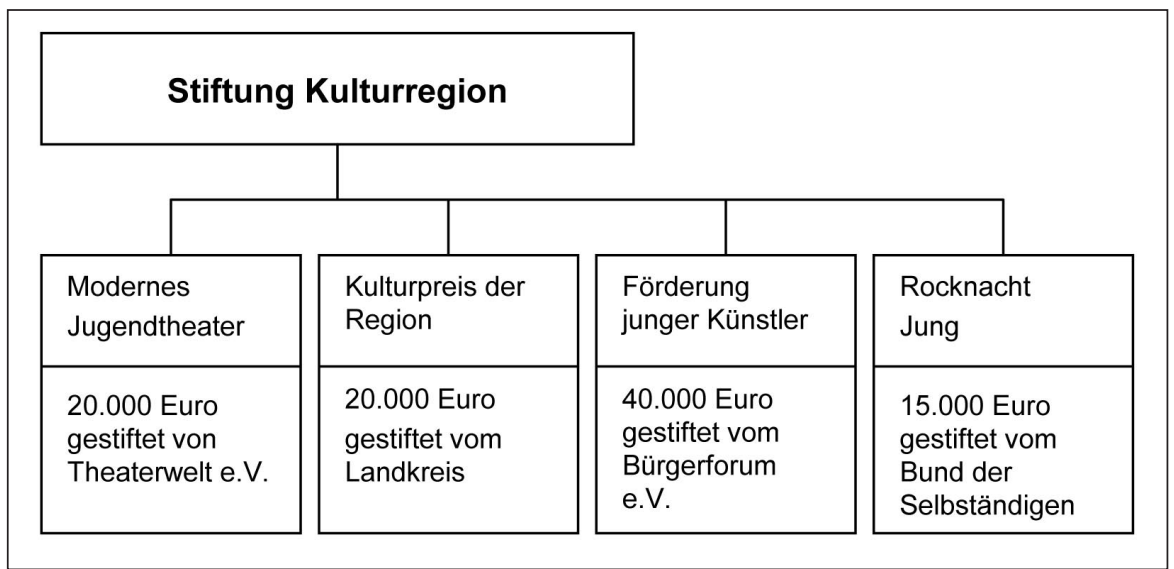

Abb. 2: Mit einer Gemeinschaftsstiftung können mehrere Ziele gleichzeitig verfolgt werden. 
sprechende Angebote zu entwickeln. Der Bund der Naturschutz in Bayern zeigt dies in einer guten Broschüre mit dem programmatischen Titel »Natur Vererben, Zukunft Stiften «.

Auch eher kleine Beträge von 5.000 oder 10.000 Euro bilden in der Summe beeindruckendes Stiftungsvermögen. Anders gesagt: Wenn es gelänge, nur ein Prozent der jährlich vererbten Vermögensmassen in gemeinnützige Stiftungen zu lenken, entstünde hier ein kapitalisierter Mehrwert von jährlich zwei Milliarden Euro, der nachhaltig und dauerhaft wirkt.

Damit von dieser Entwicklung die Anliegen kleiner gemeinnütziger Vereine profitieren, die im dritten Sektor eine Art Mittelstand gegenüber den Sozialkonzernen bilden, bietet sich hierfür das Instrument der Gemeinschaftsstiftung besonders an. Es bedeutet, dass sich mehrere gemeinnützige Organisationen auf einen gemeinsamen Stiftungszweck einigen (z. B. Förderung der Jugendkulturar- beit oder Förderung von Mehrgenerationenhäusern) und eine rechtlich selbstständige Stiftung gründen.

Die Gemeinschaftsstiftung ist also eine Stiftung mit mehreren Stiftern und $\mathrm{Zu}$ stiftern, die dem Gemeinwohl dient. Mit Stiftung wird hier sowohl der Vorgang der Widmung von Vermögen für einen oder mehrere bestimmte Zwecke bezeichnet, als auch das wirtschaftlich-rechtliche Gebilde, das als Resultat dieses Vorgangs entsteht: Im rechtlichen Sinne ist dies eine eigene Organisation, die auf Dauer Vermögen für einen bestimmten gemeinnützigen Zweck einsetzt und als rechtlich selbstständige Stiftung »sich selbst « gehört.

Die rechtlichen Voraussetzungen, die eine Gemeinschaftsstiftung erfüllen muss, sind weitgehend gleich wie die, die eine sonstige Stiftung erfüllt, die lediglich einen Stifter hat: Aus der Satzung muss klar hervorgehen, welcher Zweck angestrebt wird und wie dieser satzungsgemäße Zweck sich verwirklicht (vergleiche BGB $\mathbb{} \$ 80 \mathrm{ff}$.).
Jedoch ergeben sich einige organisatorische Besonderheiten, die bei einer Stiftung, die lediglich einen Stifter hat, nicht auftreten:

- Als Stiftungsorgan ist laut Gesetz nur der Vorstand vorgeschrieben. Wer führt bei einer Gemeinschaftsstiftung den Vorsitz? Um hier eventuellen Streitigkeiten bereits im Vorfeld zuvorzukommen, ist ein rollierendes System denkbar. Jedes Jahr oder alle zwei Jahre wechselt der Vorsitz unter den beteiligten Einrichtungen. Wem dies zu kompliziert ist, der sollte beispielsweise durch ein Kuratorium die Möglichkeit eröffnen, dass jeder beteiligte Zustifter hier mit Stimmrecht vertreten ist.

- Kein Zustifter darf bei der öffentlichen Präsentation der Stiftung benachteiligt werden. Geschehen hier Unachtsamkeiten, wird die weitere Zusammenarbeit gefährdet.

- Alle Kommunikationsmaßnahmen sollten von Anfang an auf einer gemeinsamen Plattform geplant werden,

\section{Wie man eine Gemeinschaftsstiftung erfolgreich startet}

Bevor Sie sich geeignete Partner suchen, machen Sie sich klar, dass Sie nicht auf Gedeih und Verderb viele Jahre hindurch eine strategische Allianz bilden müssen. Es ist durchaus möglich, dass ab einer bestimmten Höhe des Kapitalstocks eine Ausgründung in eine selbstständige Stiftung erfolgt, die dann ihrerseits, getragen von einem einzelnen Verein, gemeinnützige Zwecke im Gemeinwesen verfolgt.

Mit diesem pragmatischen Vorgehen wird der Erwartungsdruck, der sonst auf den künftigen Partnern lasten könnte, gemindert, die Idee des Stiftens und die Idee der Stiftung wird innerhalb einer Region verbreitet und verankert. Betrachten sie die Konzeption Ihrer Gemeinschaftsstiftung als Beitrag zu einer Kultur und Traditionsbildung des Stiftens als gesamtgesellschaftliche Aufgabe.

Die beiden folgenden Checklisten bieten Ihnen eine erste Orientierung, wie das Projekt Gemeinschaftsstiftung angegangen werden kann.

Acht Meilensteine bei der Errichtung Ihrer Gemeinschaftsstiftung

1. Wir haben in Stichworten die Zielsetzung unserer geplanten Gemeinschaftsstiftung festgelegt.

2. In unserer Region haben wir drei potenzielle Partner identifiziert.

3. Die Vorgespräche mit den Partnern werden vorbereitet und durchgeführt.

4. Es ist eine gemeinsame Konzeption erarbeitet.

5. Eine Satzung ist erstellt und vom Finanzamt und der Stiftungsaufsicht geprüft.

6. Die interne Aufgabenverteilung unter den Partnern ist festgelegt.

7. Das angestrebte Stiftungsvermögen für die nächsten fünf bis zehn Jahre ist festgelegt.
8. Es ist eine Fundraising-Strategie für die nächsten drei Jahre erarbeitet.

\section{Leitfragen zur Zeitplanung}

Beantworten Sie die folgenden sieben Fragen und beginnen Sie damit, einen groben Zeitplan zu erstellen.

1. Ab wann möchten Sie das Konzept Gemeinschaftsstiftung intern, das heißt innerhalb Ihrer Organisation diskutieren?

2. Ab welchem Zeitpunkt wird in Ihrer Organisation und den beteiligten Partnerorganisationen der Beschluss zur Errichtung einer Gemeinschaftsstiftung gefasst werden können?

3. Ab wann wird eine Projektgruppe eingesetzt, die die Pilotphase plant?

4. Zu welchem Zeitpunkt soll die Gemeinschaftsstiftung errichtet und genehmigt sein?

5. Wann sollen die Mitglieder der einzelnen Stiftungsorgane benannt werden?

6. Wann soll die Gemeinschaftsstiftung mit ihrer operativen Tätigkeit beginnen?

7. Ab wann soll die Gemeinschaftsstiftung der Öffentlichkeit vorgestellt werden?

Links, die weiterhelfen

- Die Stiftung Mitarbeit präsentiert unter http://www.wegweiser-buergergesellschaft.de ein hervorragendes Angebot. Der Menüpunkt »Praxishilfen « und dort der Button »Stiftungen « informiert knapp und umfassend.

- Die Seite der »Initiative Bürgerstiftungen « ist ebenfalls gut gemacht: http://217.91.112.229/ibsconfig/_html/grundlagen.html

Wolfgang Antes 
die jeden Partner angemessen berücksichtigt.

Auch gemeinnützige Vereine können übrigens entgegen der landläufigen Meinung langfristig Rücklagen bilden, die in ein Stiftungsvermögen überführt werden sollen (vgl. Kasten »Rücklagen bilden für Stiftungen «).
Der besondere Charme einer Gemeinschaftsstiftung besteht - wie erwähnt vor allem darin, dass sie selbst als Dachorganisation für unselbstständige Stiftungen oder Stiftungsfonds fungieren kann. Die Darstellung von unselbstständigen Stiftungen bietet als PR- und Fundraisinginstrument weitergehende Vorteile.

\section{»Ein Prozent Erbmasse generieren zwei Milliarden Euro im Jahr«}

Problematisch ist die Sache grundsätzlich, wenn es sich beim Mitstifter um eine Kommune, also um eine öffentliche Körperschaft handelt. Hier divergieren die Stiftungsgesetze der jeweiligen Bundesländer. In aller Regel darf jedoch eine Gemeinde sich in Form einer $\mathrm{Zu}$ stiftung an der Errichtung einer Stiftung nur dann beteiligen, wenn die Gemeinde einen angemessenen Einfluss, insbesondere natürlich in den entscheidenden Organen, also dem Vorstand der Stiftung erhält und diesen Einfluss durch die Vertretung der Interessen der Gemeinde geltend macht. Man wird im Einzelfall prüfen müssen, ob hier die Beteiligung einer Kommune sinnvoll ist, oder zu unüberbrückbaren Verfahrensschwierigkeiten führt.
Neben den üblichen und wichtigen Kommunikationsmaßnahmen ist es selbstverständlich möglich und sinnvoll, eine unselbstständige Stiftung mit einem eigenen Namen zu versehen. Das könnte zum Beispiel der Name eines vermögenden Einzelstifters als Privatperson oder einer Firma sein, die damit dauerhaft öffentlich benannt bleibt und immer mit dem jeweiligen Stiftungszweck kommuniziert wird. Deren Zustiftung wird der unselbstständigen Stiftung zugeordnet, die damit vielleicht einen pointierten Zweck innerhalb des Spektrums Jugendkulturarbeit übernimmt (z. B. Nachwuchsförderung). Damit lässt sich beispielsweise eine jährliche Preisverleihung verbinden.

Eine weitere interessante Möglichkeit bietet eine rechtlich nicht selbstständige

\section{Rücklagen bilden für Stiftungen}

Das Vorurteil hält sich hartnäckig, gemeinnützige Vereine seien aufgrund der gesetzlichen Bestimmung des zeitnahen Mittelverbrauchs gar nicht in der Lage sind, Kapitalsummen in ein Stiftungsvermögen überzuführen.

Hier liegt ein Missverständnis vor. Es ist zwar richtig, dass gemeinnützige Vereine grundsätzlich verpflichtet sind, ihre Mittel, die sie aus öffentlichen Zuschüssen bezogen haben, zeitnah, das heißt innerhalb eines Haushaltsjahres auszugeben, um dann für das nächste Haushaltsjahr wieder neue Mittel als Zuschuss zu erhalten. Es unterliegen jedoch nicht alle Mittel einer gemeinnützigen Organisation dem Gebot der zeitnahen Verwendungspflicht. So dürfen bestimmte Mittel steuerlich unschädlich im Vermögen gehalten werden. Dazu gehören im Einzelnen:

- Zuwendungen, die aufgrund eines Spendenaufrufs oder einer Spendenkampagne eingehen, wenn vorab eindeutig klar ersichtlich ist, dass die eingegangenen Beträge unter Bildung und Aufstockung des jeweiligen Stiftungsvermögens vorgesehen sind.

- Einzelzuwendungen, bei denen der Zuwendende ausdrücklich erklärt, dass die Zuwendung zur Erhöhung eines Stiftungsvermögens bestimmt ist.

- Zuwendungen von Todes wegen, wenn der Erblasser die Mittel nicht ausdrücklich für den laufenden Aufwand vorgeschrieben hat.

- Auch freie Rücklagen nach $\$ 58$ Nr. 7 a AO können dem Stiftungsstock zugeführt werden. Nach dieser Rechtsvorschrift darf die gemeinnützige Organisation jährlich bis zu einem Viertel des Einnahmeüberschusses aus der Vermögensverwaltung in eine so genannte freie Rücklage einstellen. Die freie Rücklage steht dann zur freien Verfügung.

Stiftung (z. B. als sogenannter Stiftungsfonds) dadurch, dass Zustiftungen geworben werden können, die ganz im Gegensatz zur normalen Gemeinschaftsstiftung oder zur normalen Rechtsform einer Stiftung teilweise wieder aufgezehrt werden können. Das bedeutet: Eine $\mathrm{Zu}$ stiftung zu einem rechtlich unselbstständigen Stiftungsfonds unterliegt nicht den gleichen gesetzlichen Bedingungen wie eine Zustiftung zu einer rechtlich selbstständigen Stiftung, die ihren Kapitalstock weitgehend erhalten muss. In der Satzung eines Stiftungsfonds lässt sich sogar festlegen, dass in einem bestimmten Zeitraum das Kapital ganz aufgezehrt wird. Das ist dann sinnvoll, wenn zum Beispiel eine bestimmte Aufgabe in einem definierten Zeitraum aller Voraussicht nach erledigt ist.

Aus den bisherigen Fundraising-Erfahrungen lässt sich schlussfolgern, dass sich der Spendenmarkt vom Stiftungsmarkt grundsätzlich unterscheidet und das nicht nur zahlenmäßig. Der Spendermarkt ist groß, teilweise anonym und regelt sich spontan. Das lässt sich daran zeigen, dass bei manchen Katastrophen, politischen Konstellationen etc. die Spendenbereitschaft sprunghaft steigt und dann wieder abflaut.

Der Stiftermarkt verhält sich anders. Wer eine kleinere oder größere Summe seines Vermögens stiftet und sich damit dieser Summe endgültig entledigt, tut das wohl durchdacht mit einer bestimmten Absicht. Er oder sie ist in der Regel gut informiert und trägt sich seit Längerem mit diesem Gedanken.

Gestützt wird diese These durch eine Untersuchung der Bank für Sozialwirtschaft. Hier wird gezeigt, dass Stiftungen ein grundsätzlich besseres Image als gemeinnützige Vereine haben. In Stiftungen angelegte Gelder gelten als wirkungsvoller und nachhaltiger angelegt als Spenden im gemeinnützigen Sektor. Diesen Imagevorteil sollten Gemeinschaftsstiftungen pflegen und sich zunutze machen. 